\title{
Indicación geográfica de productos agrícolas: situación y perspectivas para la pequeña explotación familiar en Brasil
}

\author{
Fernando Goulart Rocha \\ Instituto Federal de Santa Catarina. Departamento de Geografia \\ fernandogr@ifsc.edu.br
}

\author{
Antoni F. Tulla \\ Universitat Autònoma de Barcelona. Departament de Geografia \\ antoni.tulla@uab.cat
}

\section{Resumen}

La indicación geográfica es un mecanismo de protección para los productos vinculados a zonas geográficas específicas. Se trata de una estrategia de los productores agrarios con el fin de que la producción y comercialización de sus mercancías continúen siendo viables. La región de Vale dos Vinhedos, en el estado brasileño de Rio Grande do Sul, es la primera con indicación geográfica reconocida. Actualmente, hay treinta productos en el país con indicación de procedencia y ocho con indicación de origen. La expansión de los registros de certificación de productos agrícolas, principalmente de las pequeñas explotaciones familiares, se considera como una estrategia importante para la regeneración productiva y económica de las zonas rurales. Para los pequeños productores, la obtención de una indicación geográfica se relaciona con la buena reputación entre los consumidores de los productos certificados. En el ámbito territorial, la indicación geográfica ha contribuido a través de la gobernanza cooperativa en la creación de políticas regionales de desarrollo rural.

Palabras clave: indicación geográfica; agricultura; Brasil; desarrollo; rural.

Resum. Indicació geogràfica de productes agrícoles: situació $i$ perspectives per a les petites explotacions familiars del Brasil

La indicació geogràfica és un mecanisme de protecció per als productes vinculats a zones geogràfiques específiques. Es tracta d'una estratègia dels productors agrícoles per tal d'aconseguir que la producció i la comercialització de la seva mercaderia continuï sent viable. La regió de Vale dos Vinhedos, a l'estat brasiler de Rio Grande do Sul, és la primera amb indicació geogràfica reconeguda. Actualment, al país hi ha trenta productes amb indicació de procedència i vuit amb indicació d'origen. L'expansió dels registres de certificació de productes agrícoles, principalment de les petites explotacions familiars, es considera una estratègia important per a la regeneració productiva i econòmica de les zones rurals. Per 
als petits productors, l'obtenció d'una indicació geogràfica es relaciona amb la bona reputació dels productes certificats entre els consumidors. En l'àmbit territorial, la indicació geogràfica ha contribuït a través de la governança cooperativa a la construcció de polítiques regionals de desenvolupament rural.

Paraules clau: indicació geogràfica; agricultura; Brasil; desenvolupament; rural.

Résumé. Indication géographique de produits agricoles: situation et perspectives pour la petite exploitation familiale au Brésil

L'indication géographique est un mécanisme de protection des produits liés à des zones géographiques spécifiques. Elle est constituée par une stratégie des produits agraires afin de pérenniser la viabilité de la production et la commercialisation des marchandises. Au Brésil, le Vale dos Vinhedos, dans l'État de Rio Grande do Sul, a été la première région à avoir obtenu la reconnaissance d'indication géographique. Il y a actuellement dans ce pays trente produits avec une indication de provenance et huit produits avec une indication d'origine. L'expansion des registres de certification est considérée comme une stratégie importante dans la régénération productive et économique des zones rurales. L'obtention d'une indication géographique ouvre pour les petits producteurs des perspectives liées à la bonne réputation chez les consommateurs des produits certifiés. Au niveau territorial, l'indication géographique a contribué, grâce à la gouvernance coopérative, à la construction de politiques régionales de développement rural.

Mots-clé: indication géographique; agriculture; Brésil; développement; rural.

Abstract. Geographical Indication of Agricultural Products: The Current Situation and Prospects for Family Farms in Brazil

A geographical indication is a means of protection for products originating from specific geographical areas. It gives a specific form to a strategy enabling agricultural producers to maintain the viability of both the production and commercialization of their goods. In Brazil, the Vale dos Vinhedos in the state of Rio Grande do Sul was the first region with a recognised geographical indication. At the moment, there are thirty products in the country with geographical certificates and eight with certificates of origin. The growth in certification of agricultural products, mainly from family farms, is valued as an important strategy for the productive and economic regeneration of rural areas. The future prospects of small producers, when awarded a geographical certificate, are linked to the good reputation of certified products among consumers. In the territorial field, geographical indication has contributed through cooperative governance to the building of regional policies for rural development.

Keywords: geographical indication; agriculture; Brazil; development; rural.

\section{Sumario}

\section{Introducción}

2. Indicación geográfica: definición y marcos legales

3. Indicación geográfica de productos agrícolas en Brasil
4. Indicación geográfica en la pequeña producción familiar en Brasil

5. Consideraciones finales

Referencias bibliográficas 


\section{Introducción}

El marco general de la agricultura mundial posterior a la Revolución Verde se caracterizó por la reducción de las variedades de plantas cultivadas en favor de la productividad. La adopción de ese modelo de desarrollo finalizó en las últimas décadas del siglo pasado, amparado en el incremento de todo tipo de saber técnico aplicado a la producción agrícola. El desarrollo tecnológico aceleró los tiempos y ritmos en la agricultura, a partir de la domesticación de plantas, y aumentó la potencialidad de la actividad tal como hoy la conocemos. En este mundo rural dominado por las inversiones en maquinaria, la agricultura y «o respeito tradicional às condições naturais, cedeu lugar a um novo calendário baseado na ciência, na técnica e no conhecimento» (Santos, 1996: 207) ${ }^{1}$.

Para Paterniani (2001), las transformaciones técnicas acaecidas en la agricultura se respaldaron en una preocupación por la disponibilidad de alimentos y, en este sentido, la aplicación tecnológica y la selección de variedades de especies más aptas para el cultivo fue una respuesta a las tesis que planteaban la posibilidad de un déficit alimentario global. Con todo, para Balsan (2006), la expansión de los elementos técnicos en la agricultura expresa principalmente el poder de las grandes empresas de insumos y maquinarias directamente interesadas en la modernización de la actividad agrícola. En la misma dirección, según Graziano da Silva (1999), los avances tecnológicos permitieron reducir el período de capital adelantado a la producción, a causa de una mayor velocidad de rotación del capital, a medida que la diminución del tiempo de producción repercute directamente en la reducción del tiempo de trabajo.

A pesar de que es posible establecer pros y contras sobre los efectos de la revolución tecnológica verificados en esa etapa de desarrollo de las fuerzas productivas en el campo, es innegable que esos cambios correspondieron, en general, a la reducción de la producción familiar en el mercado, pues si la revolución tecnológica amplió la oferta de alimentos, su suministro pasó a estar concentrado en una pequeña parcela de productores capitalizados que, con el fin de viabilizar económicamente lo que producían, pasaron a especializarse en un monocultivo.

Esta condición es especialmente notable en un país de dimensiones continentales y de ocupación relativamente reciente como Brasil, en el que las tierras fueron poseídas rápidamente por el gran latifundio monocultor. En dichos latifundios, la producción agrícola estuvo y permanece dominada por los elementos tecnológicos que incluyen insumos químicos, equipos y maquinaria, definidos como los más eficientes aplicados al manejo de las labranzas y capaces de reducir el precio final de la producción. En este contexto, la dificultad de inserción comercial de la producción agrícola familiar en el mercado reside justamente en el desequilibrio de fuerzas existentes entre pequeños y grandes productores: mientras que para los grandes productores no hay dificultades en

1. «El respeto tradicional a las condiciones naturales, cedió lugar a un nuevo calendario basado en la ciencia, la técnica y el conocimiento» (N. del T.) 
la negociación de la producción, las dificultades de colocación del producto en el mercado, a un precio satisfactorio y sin la intermediación de agentes especuladores, es uno de los grandes desafíos de la agricultura familiar en Brasil.

Con el propósito de compensar tal desventaja, la venta de la producción a la agroindustria, por medio de contratos previos a la siembra (contratos de integración), es una estrategia de los pequeños productores con el fin de garantizar una renta mínima sin el riesgo de la falta de compradores. Para las empresas procesadoras, los contratos de integración son beneficiosos en la medida que por medio de ellos pueden prescindir de las áreas de producción y obtener ventajas a través de la especulación sobre el valor del bien adquirido. Por lo tanto, los grupos agroindustriales invierten en propaganda dirigida a los agricultores en que se indica que si bien las firmas integradoras pagan poco, ofrecen un mercado seguro (Paulilo, 1990).

Con el propósito de revertir la situación expuesta, las certificaciones de indicación geográfica (IG) son mecanismos que pretenden de alguna forma corregir los desequilibrios comerciales, y al mismo tiempo recuperar o valorizar un determinado producto agrícola a fin de que mantenga viable su producción. Como una estrategia de desarrollo para las regiones rurales, la IG es una acción articulada que tiene la intención de inducir cambios en el medio rural. En este contexto, la creación de productos y servicios certificados, destinados a mercados de consumo especializados, es una herramienta que pretende aumentar la capitalización y mejorar la gestión de la producción de las pequeñas explotaciones. Por otra parte, la certificación es una manera de proteger el valor de identidad de la pequeña producción, superando el paradigma de la producción agrícola a gran escala y el apoderamiento por la industria agroalimentaria del capital generado por el trabajo de las familias rurales.

Sin embargo, el éxito en el desarrollo de esta estrategia está condicionado a un proyecto de organización colectiva de los productores. Al mismo tiempo, la participación del Estado como agente normativo con el fin de fomentar, financiar y proteger los productos agrícolas con denominación de origen es esencial. Conforme Schneider (2010), la participación del Estado como agente de desarrollo rural es fundamental por ser la esfera social con la legitimidad política de proponer y conducir medidas que favorezcan los cambios en la estructura productiva de las regiones agrícolas.

En Brasil, la política estatal de certificación de productos agrícolas llevada a cabo por el Ministerio de Agricultura, Ganadería y Abastecimiento (MAPA, por sus siglas en portugués), es un ejemplo de la participación del Estado en los proyectos de desarrollo rural a nivel local. El eje principal de esta política es valorar la pequeña explotación familiar por la diferenciación de sus productos e integración comercial a través de la certificación de origen. Las oficinas del ministerio en los estados realizan diversas labores para incentivar la certificación. Primero identifican a los productos con potencial y después orientarán a los actores locales en el proceso de obtención, para que puedan incrementar los ingresos y la rentabilidad de la explotación. Paralelamente, el Programa Nacional de Fortalecimiento de la Agricultura Familiar canaliza el crédito 
bancario específicamente para financiar a los pequeños agricultores con el fin de invertir en la calificación de su producto y su procesamiento para la comercialización. Es importante resaltar que las ventajas de las IG están relacionadas precisamente con la trazabilidad, el reconocimiento de la calidad, la delimitación y la reputación positiva de un área en que los sistemas de producción y los productos resultantes son notoriamente distintos. Así, la IG se constituye como un mecanismo de protección de bienes agrícolas elaborados regionalmente y vinculados a la especificidad productiva del territorio. Este vínculo está establecido por la relación entre producto y área de producción que en virtud de la tecnología y el know-how disponibles consiguen distinguirse en el mercado frente a las demás regiones productoras (Vargas, 2008; Valente et al., 2012). A su vez, en relación con la estrategia de desarrollo, la IG se asienta sobre la decisión de los agricultores para comercializar los bienes que producen, es decir, en la ruptura de la condición marginal de estos productores al llevar a cabo una producción de capital. Para lograr este objetivo, la IG se establece como medida de valoración de la agricultura familiar, no sólo a nivel de los establecimientos, sino en términos regionales y de la economía rural en su conjunto (Kageyama, 2004; Castellano, 2002).

Considerando las características relacionadas a las IG y con el objetivo de discutir las políticas actuales de su implantación en la pequeña producción familiar en Brasil, este trabajo está organizado de la siguiente forma: primero se define y se presentan los marcos legales de las indicaciones geográficas en el ámbito internacional. A continuación se destaca la reglamentación y el escenario actual de registro de la IG de productos agrícolas en el país y se evalúa la situación actual y las perspectivas para la pequeña producción familiar a partir de la certificación de tales productos. En las consideraciones finales, se discuten las propuestas para las áreas rurales considerando el crecimiento del número de productos con IG verificados en los últimos años en el territorio brasileño.

\section{Indicación geográfica: definición y marcos legales}

De acuerdo con la Organización Mundial de la Propiedad Intelectual (WIPO), la IG es una marca que se imprime a productos con calidad, característica o distinción determinada por su origen (WIPO, 2013). Asociada directamente al territorio, la IG procura valorizar a los productores legítimos de una mercancía y su lugar de producción. Reglamentados históricamente y reconocidos por diversos países, los protocolos de IG fueron consagrados por el Acuerdo de Madrid, de 1891, y por el Arreglo de Lisboa, de 1958. Con el primero fue asegurado el registro internacional de marcas de productos registrados en uno de los países signatarios. El Arreglo de Lisboa, por su parte, trató específicamente del registro internacional de productos con denominación de origen. A través de ese acuerdo quedó aclarado que por denominación de origen se entiende la denominación de un producto originario de un país, de una región o de una localidad y cuya calidad o característica se deben exclusiva o esencialmente al medio geográfico, comprendidos los factores naturales y humanos. 
Los mecanismos de las IG se dividen en dos tipos: la Indicación de Procedencia (IP) y la Denominación de Origen (DO ${ }^{2}$. La DO comprende el tipo de IG más consagrada y conocida, y su aporte legal tiene el respaldado por la WIPO. En la literatura internacional sobre el tema es posible encontrar, todavía, términos que hacen referencia a clases de certificaciones tales como: «Denominación de Origen Controlada», "Apelación de Origen», «Indicación Geográfica Reglamentada» o "Indicación de Procedencia Controlada». De forma general, los mencionados protocolos de certificación corresponden a una de las dos categorías de certificación mencionadas (IP o DO) y están relacionadas con el grado de exigencia para la concesión de uno de dichos registros. Entre tanto, mientras los registros de DO obedecen a criterios rígidos de certificación, las indicaciones de procedencia presentan usualmente requisitos menos rigurosos para que los productos reciban algún sello de $\mathrm{IG}^{3}$.

En Europa, la decisión de la Cour d'Angers ${ }^{4}$ en $1887^{5}$ a favor de utilizar el término champagne para la designación de vinos espumosos elaborados exclusivamente en esta provincia se considera uno de los episodios más importantes para el desarrollo de la protección jurídica de los productos vinculados al territorio (Orozco Argote, 2008; Tortorelli, 2010). Sin embargo, la adopción del sistema europeo de clasificación y etiquetado de los productos de origen no llegó hasta 1970 (Calliari et al., 2007). Más recientemente, el Reglamento n. ${ }^{\circ} 1576 / 89$ y 1493/99, referente a las bebidas espirituosas y a los vinos, y el Reglamento n. ${ }^{\circ}$ 2081/92, que institucionaliza los procedimientos para la protección de las Indicaciones Geográficas y Denominaciones de Origen de los Productos Agrícolas y Géneros Alimenticios en la Comunidad Económica Europea (EEC), constituyen la base definidora de las IG en Europa.

De acuerdo con Pallet y Brabet (2005), esos reglamentos buscan resguardar la DO y, en ese sentido, actúan para que se mantenga un vínculo estrecho entre un determinado producto y el medio geográfico en el cual es obtenido. Para los productos con IP, los reglamentos establecen que la relación con el medio geográfico deberá estar prevista en por lo menos una etapa de producción. En otro sentido, se reserva a los compradores el derecho de acceso a la información sobre el producto que adquiere, considerando la tendencia creciente de consumidores que privilegian la alimentación de calidad.

Actualmente, 711 productos alimenticios europeos son certificados por la Denominación de Origen y 728 por la Indicación de Procedencia (DOOR, 2013). Entre los productos europeos con IG de origen se pueden citar bebidas como el Champagne (Francia), el Prosecco (Italia) y la sidra de Asturias (España); los quesos como el Roquefort y el Camembert de Normandía (Francia),

2. En Europa se utiliza también una tercera categoría denominada «Productos con Especialidad Tradicional Garantizada».

3. Sin embargo, muchos autores no hacen distinción alguna en el uso de los términos.

4. Para la Corte: «Se entiende por Champagne o Vino champagne el vino cosechado y elaborado exclusivamente en Champagne, antigua provincia de Francia, determinado según criterios geográficos, y cuyos límites no se puede ampliar o restringir» (CVIC, 2003: 15).

5. La sentencia judicial de 1887 se convirtió en ley en 1911 (CVIC, 2003: 25). 
el Parmigiano Regiano, el Provolone Valpadana, el Mozzarella di Bufala Campana y el Gorgonzola (Italia).

En países como Estados Unidos, Canadá y Australia, las IG y su legislación son entendidas de forma distinta y están relacionadas con el sistema de protección de marcas. En estos países, un producto puede ser certificado de forma individual o colectiva como cualquier otra característica de un producto (Herrmann, 2011). La posición norteamericana y de los países alineados con ésta (Argentina, Australia, Canadá, Chile, Colombia, Ecuador, Salvador, Filipinas, Guatemala, Honduras, Nueva Zelanda, Panamá, Paraguay, República Dominicana y Taiwán) en relación a la metodología de concesión de IG y su reputación internacional es, de esta forma, divergente del esquema europeo. Ello ha provocado en el mercado internacional la discusión sobre la protección geográfica de productos de origen, que tiene su ápice en la Ronda de Uruguay (1989-1994), cuando la Organización Mundial del Comercio (OMC) pretendió aprobar las IG como parte del Acuerdo sobre los Aspectos de los Derechos de Propiedad Intelectual relacionados con el Comercio (ADPIC).

El mencionado acuerdo permitió ofrecer una dimensión multilateral a las IG así como su reconocimiento en el comercio multilateral entre los países miembros de la OMC. Para ello, el documento definió dos niveles de protección para las IG: la protección general (artículo 22) y la protección especial para vinos y bebidas espirituosas (artículo 23). Adicionalmente, el ADPIC propuso que productos con nombres consagrados por el uso genérico no podrían ser protegidos o deberían tener su protección limitada (artículo 24). Un ejemplo de IG de uso genérico es el queso "Cheddar», que no necesariamente requiere ser producido en Cheddar, en el Reino Unido, para que pueda recibir ese nombre (OMC, 2013). El artículo 24 agrega todavía que una IG no puede recibir el mismo nombre de un producto que ya haya sido registrado como marca.

Entre tanto, la redacción de los artículos 23 y 24 no ha obtenido el consenso de los países miembros de la OMC y son los que han generado los mayores obstáculos para una efectiva receptividad internacional de los sellos de IG. Esto ocurre porque mientras para los países europeos esos dispositivos pretenden reforzar la protección de los productos certificados, aunque sea necesario avanzar en la elaboración de un registro mundial de productos legalmente protegidos, los Estados Unidos y demás miembros opositores los ven como una barrera al comercio (Oliveira, 2010). Para estos países, la monopolización de nombres consagrados por el uso común perjudica las marcas, dado que inhibe el empleo de términos de referencia conocidos por los consumidores.

Como resultado del litigio entre los miembros de la OMC sobre el contenido de los artículos 23 y 24, desde 1997 se han hecho esfuerzos multilaterales con el fin de discutir y proponer un entendimiento común sobre los procesos de registro y protección universal de bienes que poseen algún tipo de IG. En 2011, un grupo de países presentó una propuesta de modificación de ADPIC. De acuerdo a esa propuesta, el artículo 23 dejaría de tratar exclusivamente de la protección de vinos y bebidas espirituosas para tratar de la protección de productos de manera general. El artículo 24, a su vez, flexibilizaría el uso 
del nombre protegido por IG por otros países miembros, con excepción de vinos y bebidas espirituosas, siempre y cuando estén registradas como marcas o comprobadamente empleadas hace más de diez años antes de 1994. Además de lo anterior, el uso de nombres genéricos es mantenido en productos de diferentes marcas, a pesar de que puedan ser simultáneamente registrados como de IG. La aprobación de las alteraciones propuestas en la redacción de estos artículos, sin embargo, permanece hasta el momento pendiente (OMC, 2011)

\section{Indicación geográfica de productos agrícolas en Brasil}

En armonía con los dispositivos presentados en el ADPIC, la ley n. ${ }^{\circ}$ 9.279/96 reglamentó para Brasil los derechos y obligaciones relativos a la propiedad intelectual e incluye en su artículo IV la defensa de los productos con IG. Cuatro años después, la Resolución n. ${ }^{\circ} 75$ del Instituto Nacional de Propiedad Intelectual (INPI) estableció las condiciones para el registro de las IG. Según la resolución, son considerados productos y servicios con IG los legalmente depositados y reconocidos por el INPI bajo la forma de IP o DO.

En concordancia con los acuerdos internacionales sobre el tema, la legislación brasileña considera IP el nombre geográfico de país, ciudad, región o localidad, que sea reconocido como centro de extracción, producción o fabricación de determinado producto o de prestación de determinado servicio. Por otro lado, la DO se refiere al nombre geográfico de país, ciudad, región o localidad, que designe un producto o servicio cuyas calidades o características se deban exclusiva o esencialmente al medio geográfico, incluidos factores naturales y humanos (INPI, 2000).

Conviene destacar, según Bruch (2008), que la legislación brasileña, a diferencia de lo propuesto por el ADPIC, extendió la protección de IG a servicios y productos no alimentarios (como artesanías de joyas, cerámicas y productos de extracción mineral). La distribución actual de los productos nacionales certificados, por tipo de producto, está ilustrado en la figura 1:

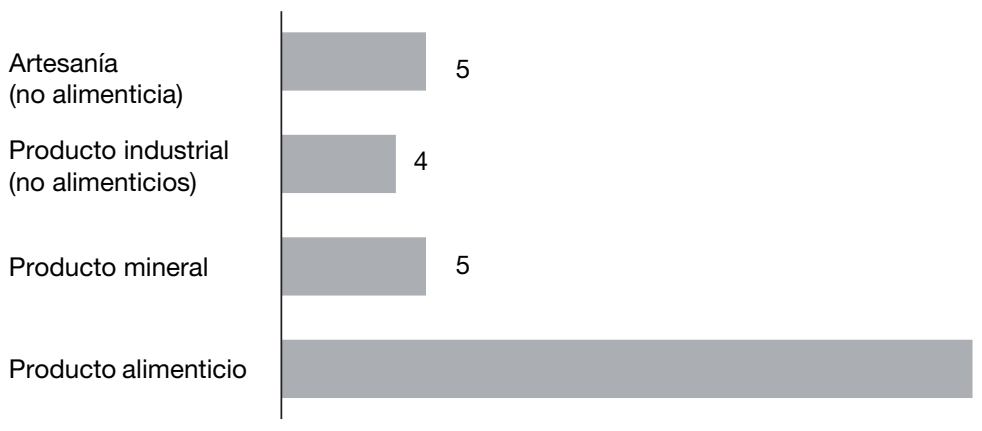

Figura 1. Indicaciones geográficas por tipo de producto en Brasil.

Fuente: elaboración propia a partir de los datos del Instituto Nacional de Propriedade Intelectual (INPI), 2013. 
Según los datos recogidos, se observa que aunque más del 60\% de los productos certificados sean alimenticios, los productos minerales, industriales y la artesanía no alimenticia representan más de un tercio de las mercancías nacionales con sello de IG. Por otro lado, a diferencia de otros países, la legislación brasileña restringió el uso del nombre geográfico. Vale recordar que el acuerdo de la OMC había permitido el uso de cualquier nombre, siempre que se hiciera mención a una localización geográfica. De esta forma, el coñac, por ejemplo, es una bebida que prescinde del uso de un complemento de indicación, ya que

Indicaciones de Procedencia:

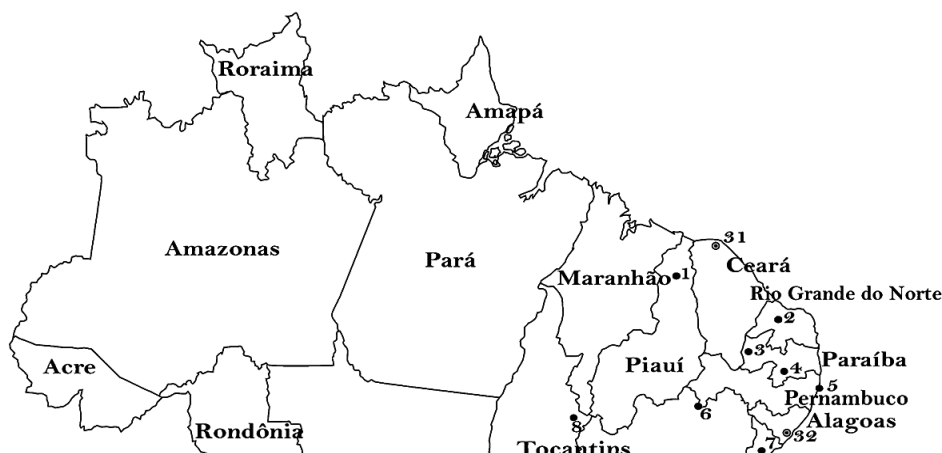

1. Ópalo precioso y joyas artesanales de l'edro II

2. Melón de Mossoró

3. Textiles de algodón colorido de Paraíba

4. Encajes del Cariri l'araibano

5. Porto digital de Recife

6. Uvas de mesa y mangas del Submedio São Francisco

7. Encajes de Divina Pastora

8. Capín dourado de Jalapão

9. Cachaça de Salinas

10. Café de la Región del Cerrado Mineiro

11. Queso de la Serra da Canastra

12. Queso Minas artesanal del Serro

19. Piezas artesanales de estaño de São João del-Rei

14. Galletas de São Tiago

15. Cafés de la Serra da Mantiqueira

16. Cacao de almendras de Linhares

17. Ollas de barro de Goiabeiras

18. Mármol de Cachoeiro de Itapemirim

19. Cafés especiales de la Alta Mogiana

20. Calzados de Franca

21. Café del Norte Pionciro do Paraná

29. Vino de la uva Goethe

23. Vinos y espumosos de Pinto Bandeira

24. Vinos de Altos Montes

25. Vale dos Vinhedos

26. Vinos de Monte Belo do Sul

27. Cuero del Vale dos Sinos

28. Dulces tradicionales de Pelotas

29. Carne de Pampa Gaúcho de la Campanha Meridional

30. Cachaça de Paraty

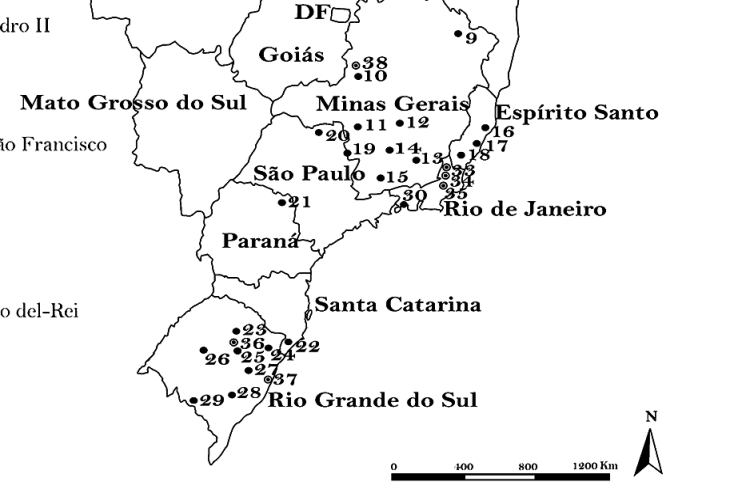

Denominaciones de Origen:

31. Camarón de la Costa Negra

32. Propóleos rojo de los manglares de Alagoas

39. Gnaisse de la Región Pedra Carijó

34. Gnaisse de la Región Pedra Madeira

35. Gnaisse de la Región Pedra Cinza

36. Vale dos Vinhedos

37. Arroz del Litoral Norte Gaúcho

38. Café de la Región del Cerrado Mineiro

Figura 2. Indicaciones geográficas brasileñas.

Fuente: elaboración propia a partir de los datos del Instituto Nacional de Propriedade Intelectual (INPI), 2013. 
está subentendido su origen: la región francesa de Cognac. En Brasil, mientras tanto, la IG debe ser explícita, aunque se subentienda el origen de un producto. Además, según la autora, la ley nacional estableció que el uso de la IG se restringe a los productores y prestadores de servicios establecidos localmente.

En Brasil, el primer proceso de reconocimiento de IG de un producto ocurrió en 2002, con la IP del Vale dos Vinhedos para vinos tintos, blancos y espumosos (Valente et al., 2012). Actualmente, en el país hay treinta productos con IP y ocho con DO (Figura 2).

En relación a la distribución espacial, los estados de Rio Grande do Sul y de Minas Gerais son los que cuentan con más productos certificados en Brasil. La trayectoria histórica de la producción agropecuaria en dichos estados, sumada a la presencia de cooperativas de productores dinámicos, a la alianza con universidades, a las empresas estatales de investigación agropecuaria y al servicio nacional de orientación sobre gestión de pequeños negocios, son las variables a las que se recurre para explicar la supremacía en el número de registros.

En términos de superficie, del número de productores o asociados ${ }^{6} \mathrm{y}$ del volumen de producción anual, las IG brasileñas también se caracterizan por la diferenciación (tabla 1).

De acuerdo con los datos expuestos, las IG nacionales poseen áreas de producción que van desde $1 \mathrm{~km}^{2}$ (IG del Porto Digital de Recife en el Sitio Histórico de la ciudad del mismo nombre) hasta $56.000 \mathrm{~km}^{2}$ (IG de los Textiles de Algodón Colorido, delimitado por la superficie total del Estado de Paraíba). El número de productores o asociados oscila a su vez de 06 (IG de los vinos y espumosos de Pinto Bandeira) hasta 7.500 (IG del Café del Norte Pioneiro do Paraná). En relación al volumen, el valor producido va desde 800-900 kg (IG del Propóleo Rojo de los Manglares de Alagoas) hasta 600 millones de toneladas (IG del Café de la Región del Cerrado Mineiro). Sin embargo, a pesar de las diferencias observadas, la participación del gobierno ha sido fundamental en la organización de estas IG. Por lo tanto, como destacó Valente y colaboradores (2012), en los últimos años ha habido esfuerzos del gobierno para que productores, comerciantes y consumidores reconozcan y valoren las indicaciones de origen.

La actuación del Estado brasileño en cuanto al fomento de la expansión de productos y servicios con $\mathrm{DO}$ ha sido notoria a partir de la reglamentación legal de 1996, cuando comenzó un movimiento creciente en el sentido de animar a los productores a la certificación de lo que producen. En este sentido, en 2005, el MAPA creó el Departamento de Propiedad Intelectual y Tecnología Agropecuaria (DEPTA). En este, desde 2006, funciona la Coordinación de Incentivo a la Indicación Geográfica de Productos Agrícolas (CIG), exclusiva para tratar la propiedad intelectual de productos provenientes de la agricultura. Esta coordinación es responsable de la expedición del instrumento oficial que

6. Los productores son miembros individuales directamente vinculados a gestión de la IG mientras que los asociados son empresas, entidades o sociedades cooperativas que representan un conjunto de productores miembros de la IG. 
Tabla 1. Superficie, número de productores o asociados y producción anual de las IG brasileñas

\begin{tabular}{|c|c|c|c|}
\hline Indicación geográfica & $\begin{array}{l}\text { Superficie } \\
\text { en } \mathrm{km}^{2}\end{array}$ & $\begin{array}{l}\text { Número de } \\
\text { productores } \\
0 \text { asociados }{ }^{1}\end{array}$ & $\begin{array}{l}\text { Producción anual } \\
\text { (estimación) }\end{array}$ \\
\hline Ópalo y joyas artesanales de Pedro II & 1.518 & 107 & * \\
\hline Melón de Mossoró & 8.340 & ** & $260.000 \mathrm{t}$ \\
\hline Textiles de algodón colorido de Paraíba & 56.496 & 35 & *** \\
\hline Encajes del Cariri Paraibano & * & 4.000 & * \\
\hline Porto digital de Recife & 1 & 130 & 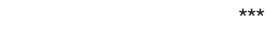 \\
\hline Uvas de mesa y mangas del Submédio São Francisco & 128 & 354 & * \\
\hline Encajes de Divina Pastora & 92 & 90 & *** \\
\hline Capín Dorado de Jalapão & 16.074 & 350 & 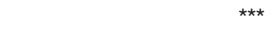 \\
\hline Cachaça de Salinas & 2.542 & 24 & $4.000 .000 \mathrm{~L}$ \\
\hline Café de la Región del Cerrado Mineiro (IP) & 1.700 & 4.500 & $300.000 .000 t$ \\
\hline Queso de la Serra da Canastra & * & * & * \\
\hline Queso Minas artesanal del Serro & * & 80 & * \\
\hline Piezas artesanales de estaño de São João del-Rei & * & * & *** \\
\hline Galletas de São Tiago & 572 & 23 & $7200 t$ \\
\hline Cafés de la Serra da Mantiqueira & 695 & 7.800 & $80.400 .000 \mathrm{t}$ \\
\hline Cacao en almendras de Linhares & 760,638 & * & $4.500 t$ \\
\hline Ollas de barro de Goiabeiras & * & 120 & 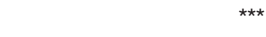 \\
\hline Mármol de Cachoeiro de Itapemirim & 1.292 & * & * \\
\hline Cafés Especiales de la Alta Mogiana & * & 23 & * \\
\hline Calzados de Franca & * & 1.015 & 26.000 .000 pares \\
\hline Café del Norte Pioneiro do Paraná & 15.567 & 7.500 & $1.300 .000 \mathrm{t}$ \\
\hline Vino de la uva Goethe & 459 & 13 & * \\
\hline Vinos y espumosos de Pinto Bandeira & 796 & 06 & * \\
\hline Vinos de Altos Montes & 173,84 & 12 & $2.000 .000 \mathrm{~L}$ \\
\hline Vale dos Vinhedos (IP) & 81,23 & 09 & $185.000 \mathrm{~L}$ \\
\hline Vinos de Monte Belo do Sul & 56,09 & 10 & $100.000 \mathrm{~L}$ \\
\hline Cuero del Vale dos Sinos & * & 60 & 45.000 .000 de piel de cuero \\
\hline Dulces tradicionales de Pelotas & * & 16 & 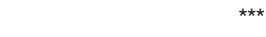 \\
\hline Carne de Pampa Gaúcho de la Campanha Meridional & 12.935 & 105 & * \\
\hline Cachaça de Paraty & 700 & 07 & 300.000 botellas \\
\hline Camarón de la Costa Negra & 429 & 33 & $9 t$ \\
\hline Propóleo rojo de los manglares de Alagoas & * & 140 & $800-900 \mathrm{~kg}$ \\
\hline Gnaisse de la Región Pedra Carijó & ** & ** & ** \\
\hline Gnaisse de la Región Pedra Madeira & ** & ** & ** \\
\hline Gnaisse de la Región Pedra Cinza & ** & ** & ** \\
\hline Vale dos Vinhedos (D0) & 72 & 10 & $545.000 \mathrm{~L}$ \\
\hline Arroz del Litoral Norte Gaúcho & 1.300 & 1.474 & $600.000 .000 t$ \\
\hline Café de la Región del Cerrado Mineiro (D0) & ** & ** & ** \\
\hline
\end{tabular}

\footnotetext{
* Datos no disponibles

** En implantación

*** Sin estimación
}

Fuente: elaboración propia a partir de los datos de: Apacs, Aicsul, Apacap, Aproarroz, Aprocam, Aprovale, Artesol, Apropampa, Ascom (2011), Doces de Pelotas, Embrapa, IBGE, Iphan, Região do Cerrado Mineiro, Sebrae, Porto Digital, Procampo (2013), Progoethe, Sindifranca. 
delimita el área geográfica de un producto a ser protegido, al mismo tiempo que ofrece aclaraciones a los productores y dispone de datos sobre los productos registrados (MAPA, 2013).

En la perspectiva de hacer accesibles los trámites para el proceso de registro como IG de un producto, el MAPA ofrece incluso gratuitamente, en la modalidad a distancia, el Curso de Propiedad Intelectual e Innovación en el Agro-negocio, además de conceder apoyo a proyectos de certificación a través de las superintendencias del ministerio en los estados. Asociado a dicho trabajo, el MAPA realizó recientemente un diagnóstico nacional de productos con potencial para la obtención de IG, y llegó a identificar 144 con condiciones de tramitar el registro.

Los centros de investigación de las universidades públicas también desempeñan un papel importante en el estudio de productos potenciales y en investigaciones que subsidien los laudos técnicos para el desarrollo de los procesos de IG. Como ejemplo, el primer producto con DO en Brasil fue el arroz producido en el litoral norte del Estado de Rio Grande do Sul (SEBRAE, 2013). La justificación para la certificación de origen consistió en que el arroz producido en esa área poseía un mayor número de granos enteros en función de la característica climática regional donde era cultivado. Esa relación fue comprobada por investigaciones realizadas por la Universidad Federal de Rio Grande do Sul que, a partir de estudios llevados a cabo en el área, emitió un documento técnico-científico decisivo para el registro de IG del cereal producido en ese Estado. En la misma dirección, la solicitud de DO del propóleo rojo con mayor contenido de isoflavonas, producido en el Estado de Alagoas, se basó en investigaciones desarrolladas por la Universidad de São Paulo.

Por su parte, el Instituto Nacional de la Propiedad Intelectual (INPI), vinculado al Ministerio de Desarrollo, Industria y Comercio Exterior (MDIC), así como el Servicio Brasileño de Apoyo a Micro y Pequeñas Empresas (SEBRAE), colaboran para que los productores conozcan y se organicen con el fin de solicitar algún tipo de IG para su producto. En este sentido, el INPI, órgano responsable del análisis de procesos y registro de IG en Brasil, publica en colaboración con el SEBRAE manuales con informaciones prácticas sobre el tema. El SEBRAE posee incluso líneas de crédito específicas para apoyar las regiones brasileñas con sello o que habían pedido un registro de IG en el INPI.

Otras instituciones, como la Empresa Brasileña de Investigación Agropecuaria (EMBRAPA, por sus siglas en portugués) y las secretarías de agricultura en los estados, asesoran a las regiones productoras a través del diagnóstico, zonificación agrícola e investigación aplicada con vistas a la certificación de productos. En las regiones que ya poseen el registro de IG, esas instituciones son fundamentales para la mejora de la calidad de la producción y para la divulgación de ese conocimiento entre los productores locales. El resultado de la participación de estos organismos en la motivación de los productores para la certificación de sus productos es el aumento en el número de registro de IG en el país. 


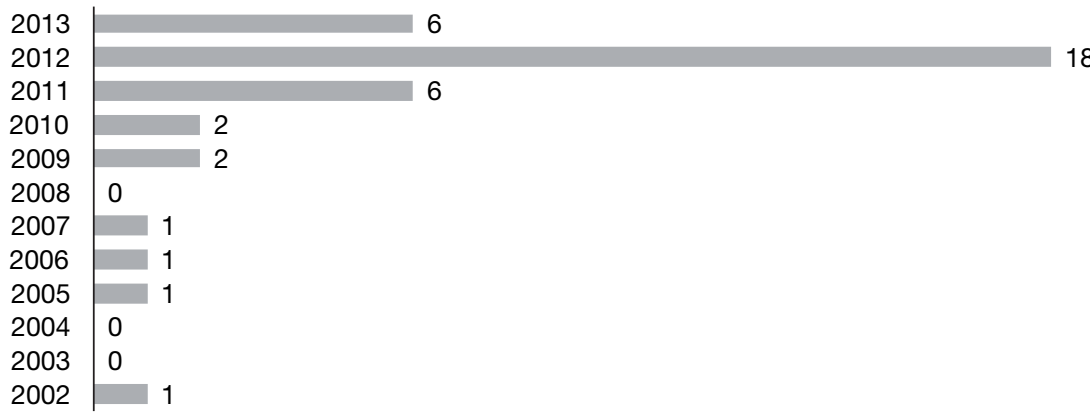

Figura 3. Evolución de los registros de IG en Brasil para los productos nacionales por año. Fuente: elaboración propia a partir de los datos del Instituto Nacional de Propriedade Intelectual (INPI), 2013.

De acuerdo con los datos de la figura 3, es posible mostrar el crecimiento en el número de registros de IG en Brasil en los últimos años, especialmente a partir de 2011, cuando se expandió la participación de las agencias estatales en el asesoramiento a las asociaciones y cooperativas de productores para obtener dos sellos de IP y DO. Actualmente, en las regiones que ya poseen registradas las IG, estas instituciones son fundamentales para mejorar la calidad de los productos y la difusión de los conocimientos de gestión y comercialización de la producción entre los agentes locales. Además de los esfuerzos institucionales, la valorización y la diferenciación comercial de los productos certificados es probablemente el principal reto al que se enfrenta Brasil, para generar una mayor motivación de los productores del reconocimiento de su producción como IG (Valente et al., 2012; Velloso, 2010). De esta forma, los esfuerzos para la obtención de sellos de IG, actualmente, han sido motivados sobre todo por la exportación. En el mercado interno, de hecho, no hay, hasta el momento, preferencia del consumidor por productos con registro de procedencia o de origen. Entre tanto, conforme anota Alonço (2005), productos como el arroz, por ejemplo, para el cual los consumidores brasileños toman la marca antes que la variable precio como referencia para la compra, es posible especular que la IG podría convertirse en un diferencial de competitividad.

\section{Indicación geográfica en la pequeña producción familiar en Brasil}

La comercialización de la producción es uno de los desafíos de las familias rurales que se dedican a las actividades agropecuarias en Brasil. A pesar de las excepciones, la pequeña producción familiar en el país ocupa una posición marginal en el abastecimiento del mercado minorista de alimentos, particularmente en lo que tiene que ver con la comercialización directa sin que exista cualquier tipo de intermediación. Los motivos que llevan a las dificultades de colocación en el mercado de productos agrícolas por los propios productores son relativamente obvios y están relacionados entre sí: la mayoría de las veces, el motivo más 
evidente se refiere a la capacidad limitada de los productores para administrar la producción. Se entiende por gestión de la producción en la actividad agropecuaria, por ejemplo, la utilización de herramientas que permitan conciliar el calendario de cosecha o de sacrificio con períodos previos de contacto con potenciales compradores, para así reducir las existencias, principalmente de productos altamente perecederos como frutas, legumbres y hortalizas. En otros casos, la gestión de la producción puede significar justamente lo contrario: mantener existencias reguladoras de forma tal que se manejen los precios en función del control de las variables de oferta y demanda. Otro instrumento de gestión está asociado a la habilidad de los productores para agregar valor al producto de su trabajo y así venderlo semiprocesado e incluso transformado.

Una segunda causa para la dificultad de inserción de la pequeña producción familiar en el mercado se debe a la fragilidad o incluso a la ausencia de asociaciones cooperativas en determinadas actividades y espacios rurales. En las áreas de producción agropecuaria en las que las asociaciones cooperativas son activas, contribuyendo a la gestión de la actividad y la capitalización de los productores, su papel en el desarrollo local es decisivo ${ }^{7}$. Para el pequeño productor, las organizaciones cooperativas no sólo permiten elevar las ganancias, debido al procesamiento, almacenamiento, reventa y distribución del producto, sino que además algunas de ellas actúan en el sentido de disminuir costos de producción y actuar para aumentar la calidad del bien producido.

Otros motivos de las dificultades de comercialización de la pequeña producción familiar en el mercado brasileño están asociados a la rígida legislación sanitaria del país que, a pesar de su indiscutible importancia, encarece el procesamiento artesanal de determinados productos y no hace viable el comercio de otros, especialmente in natura de origen animal. Adicionalmente, cabe mencionar el papel cada vez más incisivo de la industria alimentaria y de las agroindustrias, que a través de la integración vertical transforman los pequeños productores en asalariados rurales. Este tipo de relación retrae notablemente la perspectiva de colocación directa de lo producido por los pequeños productores en el mercado, pues la producción familiar tiene poca importancia en las etapas de la producción agroindustrial y en la manera como reproduce su capital (Pizzolatti, 1996).

En otros casos, los productores que resisten o no son agregados a la línea de producción agroindustrial sufren, además, por no ser capaces de generar economías de escala, y pierden eficiencia y competitividad en el mercado (Guanziroli, 2003). Una expresión de dicha pérdida de competitividad es el precio más elevado de los bienes producidos en las pequeñas unidades, sobre todo si no presentan diferencias cualitativas. Éstas se entienden como la producción agrícola o pecuaria con alguna distinción frente a las demás, ya sea por la variedad producida, por la forma de manejo o escala de producción, y es un mecanismo eficiente para que la pequeña producción agrícola pueda

7. En Brasil existen ejemplos exitosos de cooperativas de pequeños productores en actividades como el cultivo de arroz, de la uva y la producción de leche. 
destacarse comercialmente. Dicho mecanismo es utilizado, por medio de las indicaciones de origen, como base para la protección en el mercado de la producción familiar y como instrumento de mejora de las condiciones de vida de la población rural. Es por ello que, el Reglamento n. ${ }^{\circ}$ 2081/92 del Consejo de la Comunidad Económica Europea (EEC), relativo a los procedimientos para Indicaciones Geográficas y Denominaciones de Origen de los Productos Agrícolas y Géneros Alimenticios, menciona que tales productos pueden volverse una ventaja para el mundo rural, sobre todo en las regiones menos favorecidas, contribuyendo al aumento de la rentabilidad de los agricultores y su permanencia en las áreas rurales.

Adicionalmente, según Glass y Castro (2009: 11), «produtos com indicações geográficas exercem grande apelo sobre o consumidor, atraído tanto por aquela característica quanto pela qualidade» ${ }^{8}$. En este sentido, la IG puede ser una estrategia en favor de las regiones de pequeña producción familiar en la medida que invierte la lógica de la acumulación a gran escala, por la de especialización y notoriedad (Chaddad, 1996). En Brasil, el cultivo de uvas para la producción de vinos de gama alta, el manejo de variedades de arroz con menor número de granos quebrados y la crianza de camarones con niveles elevados de proteína y textura diferenciada, son ejemplos de productos de la actividad rural familiar que se insertan en el mercado nacional e internacional certificados y reconocidos por su origen. La certificación de la producción acredita y da visibilidad a la calidad de esos productos, y vuelve a posicionarse positivamente en una nueva plataforma de cotización y aceptación comercial. Además, repercute en la predisposición a evaluar positivamente los productos agrícolas por el consumidor. Sobre este aspecto, conforme Herrmann (2011), la investigación realizada por Van Ittersun y otros (2007), concluye que la percepción de los consumidores europeos hacia productos con etiquetas de certificación regional es favorable. Resultados equivalentes fueron presentados por Teuber (2011) en relación al consumo de sidra con DO por parte de los alemanes. En el mismo sentido, el estudio de Barham (2003) reveló que productos agrícolas con indicación de origen y comercializados en pequeña cantidad alcanzan hasta un 30\% más de rentabilidad en el comercio mundial de alimentos y bebidas.

Aparte de las ventajas competitivas relacionadas con el consumo, los sellos de IG contribuyen a la organización de los productores para así asegurar la calidad del bien producido y conservar la reputación del mismo en el mercado. Por lo tanto, la concesión de registros de IG está condicionada a la existencia de un consejo regulador, instancia responsable de promover y preservar la distinción atribuida al producto por los organismos certificadores (INPI, 2006; Rocha y Tulla, 2013). Sobre el funcionamiento de los consejos reguladores en Brasil, de acuerdo con el MAPA (2013: 08) «não existe uma definição clara de como ele deve ser formado. Sugere-se a inclusão de representantes

8. «Productos con indicaciones geográficas son reclamadas por el consumidor, atraído tanto por aquella característica como por la calidad» (N. del T.) 
das universidades, de instituiçōes afetadas pelo produto, dos consumidores e dos próprios produtores»?

En Brasil, debido a la baja valorización que los productos con IG poseen en el mercado, los consejos reguladores han asumido principalmente, en esa etapa de certificación de productos agrícolas, la tarea de divulgar los sellos de distinción obtenidos a través de la IG así como el control de los sistemas de producción. En este sentido, así como sucede en Europa, las IG brasileñas han apostado recientemente por la promoción turística de las IG con el objetivo de que los visitantes conozcan el proceso de producción y se amplíe el interés del consumidor por ese tipo de producto. Sin embargo, a diferencia de la experiencia europea, las iniciativas en Brasil son aún poco relevantes en términos de propuestas y resultados.

Entre tanto, se espera que los consejos reguladores, además de promover y supervisar el funcionamiento del proceso de producción, puedan también incluir a los productores con el propósito de que se constituyan como un instrumento importante de organización colectiva. Así, se espera que además de estar involucrados en la creación de normas y procedimientos de regulación de la producción certificada, puedan también operar pensando políticas y estrategias de desarrollo colectivas. Se entiende por estrategias de desarrollo, en este contexto, no sólo la valorización del producto en sí, sino además la concepción de medidas que posibiliten que la IG de un producto revierta en la mejora de las condiciones de vida de sus productores. Adicionalmente, tales estrategias se refieren a la superación de adversidades coyunturales a partir de la formulación de instrumentos de planificación a corto, medio y largo plazo. Es en este contexto que la IG, a través de su consejo regulador, puede actuar en sintonía con las políticas locales de desarrollo, sobre todo dirigidas a las áreas rurales.

Por otra parte, la IG puede convertirse en una estrategia importante para la recuperación productiva de las regiones rurales de agricultura familiar. En dichas regiones, en donde la actividad agrícola se retrae debido a las dificultades de colocación en el mercado de sus productos, a la baja cotización del producto o al ascenso de otras actividades más rentables económicamente para los productores, la IG puede ser un mecanismo de desarrollo a través de la apertura de canales especializados para el comercio de la producción. Una experiencia de ese tipo fue constatada en la recuperación del cultivo de manzanas de sidra en el Principado de Asturias (Rocha y Tulla, 2013; Fernández, 2001, Requejo, 1986).

En esta comunidad autónoma, la supremacía de la ganadería en detrimento de la agricultura tuvo su inicio a mediados de la década de 1960 y estuvo relacionada, según Fernández (2001), con el crecimiento económico y el ascenso de la demanda de productos de origen animal por parte de la población española concentrada en los grandes centros urbanos a partir de esta fecha. Debido a la expansión de la ganadería bovina, la producción de manzanas de sidra

9. «No existe una definición clara de cómo este debe ser formulado. Se sugiere la inclusión de representantes de las universidades, de instituciones afectadas por el producto, de los consumidores y de los propios productores». (N. del T.) 
retrocedió severamente hasta mediados de la década de 1990, lo que contribuyó a que parte de las áreas utilizadas para el cultivo de manzanos pasara a ser para el pastoreo o para producción de forrajes.

Sin embargo, durante la década de 1990, hubo un fortalecimiento de las asociaciones de productores de manzanas y de sidra, así como de la intervención estatal a través de la concesión de subsidios para nuevas plantaciones y la ampliación de los esfuerzos del Servicio Regional de Investigación y Desarrollo Agroalimentario (SERIDA) para la inserción competitiva del producto en el mercado. De este proceso resultó, en 2003, el registro de Denominación de Origen Protegida (DOP) de la sidra producida en Asturias. Además de proteger la bebida fermentada producida en esa comunidad autónoma, la DOP de la sidra exigió que la producción de sidras certificadas hiciese uso de manzanas producidas exclusivamente en Asturias.

Esta exigencia, como resultado del sello DOP, amplió la necesidad de obtener manzanas cultivadas localmente, por parte de los productores de sidra. A consecuencia del aumento de la demanda del producto, se percibió un alza en los precios pagados a los productores de manzanas que cultivan las variedades recomendadas para la producción de sidra con sello $\mathrm{DOP}^{10}$, lo que ocasionó volver al cultivo de manzanas por productores ${ }^{11}$ que habían abandonado dicha actividad. La experiencia asturiana en la recuperación de la producción de manzanas de sidra, a través de la DOP, evidencia cómo la IG de un producto puede ser utilizada para la revitalización económica regional y en favor de las familias que lo producen. Además, permite ver que la IG, como estrategia de desarrollo territorial, debe prever la articulación de la cadena de producción, procesamiento y comercialización del producto certificado a fin de que pueda efectivamente garantizar la demanda y la rentabilidad de los productores rurales que pretendan volver a invertir en esta actividad.

En Brasil, la IG de la cachaça producida en Paraty, en el Estado de Rio de Janeiro, es igualmente una experiencia exitosa de cómo el sello de IP puede actuar para recuperar la actividad agropecuaria. De acuerdo con Druzian y Nunes (2012: 421), «o benefício foi além do resgate da produção que quase desapareceu ao longo dos anos, pois atualmente os produtores vendem quase toda a produção aos turistas» ${ }^{12}$.

La recuperación o conservación de la producción artesanal y en pequeña escala en Brasil parece ser una tendencia cuando se analiza los estudios reali-

10. Desde 2007, las variedades de manzanas para la producción de sidra con DOP han mantenido cotizaciones de venta superiores a las variedades de mesa, y han alcanzado, en 2011, un precio medio $22 \%$ superior. En dicho año, mientras las variedades de manzanas de sidra estaban siendo revendidas a $0,37 € / \mathrm{kg}$, las de mesa eran comercializadas a $0,30 € / \mathrm{kg}$ (CRDOP, 2013).

11. En 2003 eran 171 las plantaciones registradas como cultivos de manzana con origen protegido; el 2012 ese número pasó a 270 (CRDOP, 2013).

12. «El beneficio fue más allá del rescate de la producción que casi desapareció con el paso de los años, pues actualmente los productores venden casi toda la producción a los turistas». (N. del T.) 
zados por el MAPA de productos potenciales para la obtención de IG. En el informe del Ministerio, que menciona productos potenciales para el registro de IG, aparecen el pinhão, semilla comestible del Pinheiro do Paraná ( $A$. angustifólia Bert.), el açaí, fruto del Açaizeiro (E. oleracea Mart.), el urucum (B. orellana L.) y el azafrán (C. longa L.). Los mencionados productos, a pesar de estar vinculados a los respectivos espacios rurales regionales, comparten actualmente dificultades inherentes a la pequeña producción y, en esos términos, no es segura la permanencia de productores dedicados a tales actividades sin que haya alguna intervención en el corto y medio plazo, en el sentido de protegerlas.

Es evidente que la IG por sí sola no soluciona las dificultades de comercialización de la pequeña producción familiar, y tampoco significa el reconocimiento inmediato de la calidad de los productos de origen, sobre todo en un país donde este tipo de distinción todavía no está incorporada a los criterios de referencia para el consumo por parte de la población en general. En cambio, las actividades ligadas al turismo han sido aprovechadas como medio de publicitar el producto asociado a las características de la región productora. En este contexto, las IG están presentes, frecuentemente, en la promoción de ferias, fiestas, exposiciones, demostración de campo y degustación del producto en bares, restaurantes, cafés, hoteles y posadas, especialmente en el área en que coincide con su límite territorial.

Además de promover el producto certificado, el turismo se convierte muchas veces en una estrategia de diversificación de la renta de la pequeña explotación agraria y de incremento de recursos en las regiones productoras más pobres. El registro de IG de las ollas de Cerámica de Goiabeiras, en la periferia de Vitória, capital del Estado de Espírito Santo; y de los Encajes de la Región del Cariri, en el interior del Estado de Paraíba, son ejemplos de áreas que buscan a través de la actividad productiva tradicional y del turismo redimensionar los proyectos de desarrollo local y regional. En esta dirección, el turismo se coloca como precursor de la reputación positiva del producto y de la región productora en el mercado, en cuanto la IG es un mecanismo empleado con la intención de reiterar la notoriedad conquistada por el producto.

En Brasil, la IG de los vinos del Vale dos Vinhedos (figura 4) y de Pinto Bandeira, en el Estado de Rio Grande do Sul, o la Cachaça de Salinas, en el Estado de Minas Gerais, son ejemplos de productos cuya reputación fue inicialmente consolidada por el turismo y después a causa del sello de certificación de calidad.

En la Región del Vale dos Vinhedos, la implantación de la IG resultó en la duplicación de la producción de vino y el número de turistas en 2012 fue tres veces superior al registrado en años anteriores (Druzian y Nunes, 2012; Fernández, 2012). De esta forma, es innegable que la concesión de la IG ha elevado el nivel de gobernabilidad de las regiones productoras en relación a la calidad del producto y su inserción comercial, al tiempo que ha reforzado el papel competitivo de las áreas de pequeña producción familiar en el territorio brasileño. 


\section{DENOMINAÇÃO DE ORIGEM D.O. VALE DOS VINHEDOS}

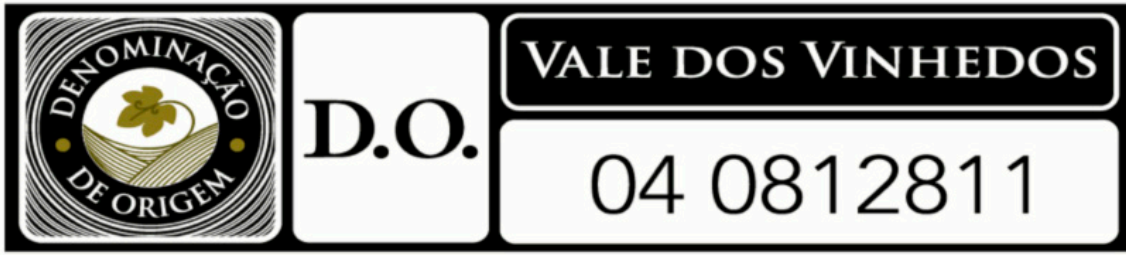

Figura 4. Sello de Indicación Geográfica tipo Denominación de Origen del Vale dos Vinhedos.

Fuente: EMBRAPA (2014).

\section{Consideraciones finales}

Aunque los protocolos internacionales de IG hayan sido firmados desde los últimos años del siglo XIx, hasta el año 2002 no fue concedido el primer registro de IP de un producto en Brasil. Actualmente, 37 productos nacionales tienen certificados de IG, de los cuales 30 son reconocidos por la procedencia y 7 por el origen. El reducido número de registros de productos con IG en Brasil contrasta con países como España, que posee 164 productos registrados; Francia, con 200 productos certificados; y Italia, con 254 (DOOR, 2013).

Las políticas recientes del MAPA apuntan, no obstante, hacía el avance de la IG de productos agrícolas brasileños, sobre todo en áreas de pequeña producción familiar. En concordancia con las directrices europeas para el desarrollo de las regiones rurales, la IG es percibida en Brasil como una estrategia de inserción de los pequeños productores en el mercado y de permanencia de esa población en el campo. Además, es una forma que se juzga eficiente en el propósito de elevar el precio de los alimentos producidos por las unidades de producción familiar. En este sentido, según Druzian y Nunes (2012), la producción de vinos con IG del Vale dos Vinhedos, en el Estado de Rio Grande do Sul, ascendió hasta un 300\% en diez años. A su vez, la carne de Pampa Gaúcho, con la obtención de la IG, pasó a tener un precio de hasta un 30\% más en relación a la que no poseía registros de procedencia geográfica.

Por un lado, para los pequeños productores, las perspectivas con la obtención de la IG están relacionadas con la reputación positiva por parte de los consumidores de los productos agrícolas certificados. La certificación potencia la competitividad de la producción en el mercado y favorece la cualificación de los actores involucrados con la producción y de gobernabilidad cooperativa del territorio. Sin embargo, la certificación de productos agropecuarios está asociada a políticas más amplias de desarrollo, las cuales presuponen proteger 
los pilares de la economía agrícola local y subsidiar medios para la proyección comercial de la producción en diferentes escalas de mercado.

Por otro lado, en Brasil, una política nacional de registro de IG de productos oriundos de la pequeña producción familiar señala la ruptura de las inversiones del estado aplicadas mayoritariamente a las áreas de agricultura de commodities, como ha sido habitual hasta la década de 1990 (Rocha, 2012). La orientación de parte de estas inversiones hacia la promoción y distinción de los productos agropecuarios de pequeña producción familiar permite mostrar, hoy, alguna preocupación por las distorsiones ocasionadas por las desigualdades verificadas en el desarrollo desigual de las regiones rurales en el país. Así, por medio de la política basada en el desarrollo endógeno de las regiones rurales, actualmente se busca valorizar la pequeña producción familiar que históricamente, a pesar de los recursos escasos, se mostró extremadamente capaz de superar las adversidades económicas.

En este contexto, se espera que la protección y certificación de productos agrícolas en Brasil contribuya a superar las adversidades más comunes aún asociadas a la pequeña producción familiar en el país: el atraso tecnológico y la frágil competitividad del mercado. A pesar de que los productos hasta ahora certificados poseen notoriedad y se distinguen positivamente entre los consumidores nacionales, se esperan nuevos avances que favorezcan la calidad y contribuyan a ampliar su comercialización, especialmente en el ámbito internacional. Para los productos con menor aceptación en la comercialización, se espera que la IG contribuya a incentivar a sus productores hacía el perfeccionamiento de las etapas de producción y procesamiento, con el fin de que puedan valerse de las ventajas añadidas de la titularidad del registro de IG. Finalmente, se espera que la IG intensifique la organización colectiva de los pequeños productores y colabore al crecimiento del empleo y la rentabilidad económica de las explotaciones agrarias en las regiones rurales brasileñas.

\section{Referencias bibliográficas}

AICSUL. <http://www.aicsul.com.br> [consulta: 11 de abril de 2014].

Alonço, Airton dos Santos (2005). Consumo, mercado e comercialização do arroz no Brasil. Pelotas: Embrapa Clima Temperado.

APACAP. <http://www.apacap.com.br> [consulta: 11 de abril de 2014].

APACS. <http://www.apacs.com.br> [consulta: 12 de abril de 2014].

APROARROZ. <http://www.aproarroz.com.br> [consulta: 11 de abril de 2014].

APROCAM. <http://www.aprocam.com.br> [consulta: 14 de abril de 2014].

APROVALE. <http://www.valedosvinhedos.com.br> [consulta: 14 de abril de 2014].

APROPAMPA. <http://www.carnedopampagaucho.com.br> [consulta: 14 de abril de 2014].

ARTESOL. <http://artesol.org.br> [consulta: 12 de abril de 2014].

ASCOM (2011). Com apoio da UFSJ, biscoitos de São Tiago podem ganhar indicação geográfica. Portal de Notícias da Universidade Federal de São João Del-Rei. <http://pgadm.ufsj.edu.br/noticias_ler.php?codigo_noticia=2719> [consulta: 12 de abril de 2014]. 
Balsan, Rosane (2006). "Impactos decorrentes da modernização da agricultura brasileira». Campo-Território, 1 (2), 123-151.

BARHAM, Elizabeth (2003). "Translating terroir: the global challenge of french». Journal of Rural Studies, 19 (1), 127-38. <http://dx.doi.org/10.1016/S0743-0167(02)00052-9>

Bruch, Kelly Lisandra (2008). Indicações geográficas para o Brasil. Século Novo: Flores da Cunha.

Calliari, Maria Alice; Buainain, Antonio Marcio; Carvalho, Sergio Medeiros Paulino de; Chamas, Claudia Ines; Salles-Filho, Sérgio Luiz Monteiro y Silveira, José Maria Ferreira Jardim da (2007). Proteção às indicações geográficas: a experiência brasileira. Seminário Latino-Iberoamericano de Gestion Tecnológica: Buenos Aires.

Castellano, Flora de Oliveira (2002). Condiçôes para ampliação da comercialização de produtos orgânicos da agricultura familiar na Grande Florianópolis/SC: estudo sobre a organização de uma central de reuniāo e distribuição de produtos. Monografia no publicada. Florianópolis: Universidade Federal de Santa Catarina.

CCPI (2013). Melão do Rio Grande do Norte pode ganhar indicação geográfica. Comissão Comissão Permanente de Propriedade Intelectual. Viçosa: Minas Gerais. <http://www.cppi.ufv.br/pt-BR/noticia/melao-do-rio-grande-do-norte-podeganhar-indicacao-geografica> [consulta: 15 de abril de 2014].

Chaddad, Fabio Ribas (1996). «Denominações de origem controlada: um projeto de pesquisa». Caderno de Pesquisas em Administração, 1(1), 1-6.

CIVC (2003). Les clés des vins de Champagne. L'appellation Champagne. Comité Interprofessionnel du Vin de Champagne. <http://www.maisons-champagne.com/pdf/appellation.pdf> [consulta: 13 de abril de 2013].

CRDOP (2013). "El Consejo en cifras: cuaderno nuestros temas». Revista del Consejo Regulador de la DOP Sidra de Asturias. Villaviciosa, 4 (1), 10-15.

Doces de Pelotas. <http://www.docesdepelotas.org.br> [consulta: 15 de abril de 2014].

DOOR. <http://ec.europa.eu/agriculture/quality/door/list.html> [consulta: 16 de octubre de 2013].

Druzian, Janice Izabel y NunEs, Itaciara Larroza. «Indicações geográficas brasileiras e impactos sobre bens agrícolas e/ou industriais». Geintec, 2 (4), 413-426.

EMBRAPA. <http://www.cnpuv.embrapa.br> [consulta: 15 de abril de 2014].

EMBRAPA (2014). Indicaçôes geográficas de vinhos finos no Brasil. Embrapa Uva e Vinho. Bento Gonçalves. <http://www.cnpuv.embrapa.br/tecnologias/ig/> [consulta: 15 de abril de 2014].

FERnÁndeZ, Jesús Arango (2001). "Agricultura y espacio rural en Asturias». Revista Asturiana de Economía, Extra 1, 69-107.

Fernandéz, Maria Gabriela Vázquez (2012). Indicaçôes geográficas e seus impactos no desenvolvimento dos pequenos produtores do Vale dos Vinhedos, RS. Tesis de Máster no publicada. Brasília: Universidade de Brasília.

Glass, Rogério Fabrício y Castro, Antônio Maria de (2009). As indicações geográficas como estratégia mercadológica para vinhos. Brasília: Embrapa.

Graziando Da Silva, José (1999). O novo rural brasileiro. São Paulo: UNICAMP.

Guanziroli, Carlos (2003) «O porquê da reforma agrária». Econômica, 4 (1), 5-24.

Herrmann, Roland (2011). «La economía de la protección de las indicaciones geográficas». Lima: Simposio Mundial sobre Indicaciones Geográficas. 
IBGE. <http://www.cidades.ibge.gov.br/xtras/home.php> [consulta: 16 de abril de $2014]$.

INPI (2000). Resolução 75: estabelece as condiçôes para o registro de indicaçóes geográficas. Rio de Janeiro: Instituto Nacional de Propriedade Industrial. Ministério do Desenvolvimento, Indústria e Comércio.

INPI (2013). Indicações Geográficas Reconhecidas. Instituto Nacional de Propriedade Industrial. Ministério do Desenvolvimento, Indústria e Comércio.

<http://www.inpi.gov.br/images/docs/lista_com_indicacoes_geograficas_concedidas__-_31-12-2013.pdf> [consulta: 12 de enero de 2013].

IPHAN. <http://portal.iphan.gov.br/> [consulta: 15 de abril de 2014].

Kageyama, Angela (2004). "Desenvolvimento rural: conceito e medida». Cadernos de Ciências \& Tecnologia, 21 (3), 379-408.

KrüCKen-Pereira, Lia (2001). O processo de valorização de produtos alimentícios através de denominações de origem e qualidade: uma abordagem de gestão do conhecimento. Tesis de máster no publicada. Florianópolis: Universidade Federal de Santa Catarina.

MAPA (2013). Guia para solicitação de registro de indicação geográfica para produtos agropecuários. Brasília: Coordenação de incentivo à indicação geográfica de produtos agropecuários. Ministério da Agricultura, Pecuária e Abastecimento.

OliveirA, Joana de Moura Leitão Barros (2010). Denominaçôes de origem e indicaçôes geográficas: proteção e impacto socioeconômico. Monografía no publicada. Lisboa: Instituto Superior de Economia e Gestão.

OMC (2011). Propuesta de proyecto de decisión del consejo de los ADPIC sobre el establecimiento de un sistema multilateral de notificación y registro de las indicaciones geográficas de vinos y bebidas espirituosas. Organización Mundial del Comercio. $<$ TN/IP/W/10/Rev.4> [consulta: 16 de octubre de 2013].

OMC (2013). ADPIC indicaciones geográficas; antecedentes y situación actual. <http://www.wto.org/spanish/tratop_s/trips_s/gi_background_s.htm> [consulta: 1 de octubre de 2013].

Orozco Argote, Iris del Rocío (2008). «Las denominaciones de origen en México». Actualidade Juridica, 17, 385-402.

Palet, Dominique y Brabet, Catherine (2005). «Os selos oficiais de qualidade dos alimentos na França e na Europa». En: Lagares, Lea; Lages, Vinicius y Braga, Christiano (coord.). Valorização dos produtos com diferencial de qualidade e identidade: indicação de qualidade e certificaçôes para competitividade nos negócios. Brasília: SEBRAE.

Paterniani, Ernesto (2001). «Agricultura sustentável nos trópicos». Estudos Avançados, 15 (43), 303-326.

<http://dx.doi.org/10.1590/S0103-40142001000300023>

Paulilo, Maria Ignês Silveira (1990). Produtor e agroinduistria: consensos e dissensos. Florianópolis: Universidade Federal de Santa Catarina.

Pizzolatti, Roland Luiz (1996). Os pequenos produtores do oeste catarinense: integrados ou entregados? Tesis doctoral no publicada. São Paulo: Universidade de São Paulo.

PORTO DIGITAL. <http://www.portodigital.org> [consulta: 17 de abril de 2014].

PROCAMPO. Em defesa da cacauicultura. Entrevista con Maurício A. Buffon, presidente da ACAL, obtenida en 08.01.2014 [en línea].

<http://www.revistaprocampo.com.br/ver-entrevista/24> [consulta: 17 de abril de 2014].

PROGOETHE. <http://www.progoethe.com.br/> [consulta: 16 de abril de 2014]. 
Região do Cerrado Mineiro. <http://cerradomineiro.org> [consulta: 15 de abril de 2014].

Requejo, Sergio Alvarez (1986). La sidra en Asturias. Villaviciosa: SERIDA.

Rocha, Fernando Goulart (2012). Cultivo de arroz irrigado na Região Sul de Santa Catarina. Florianópolis: IFSC.

Rocha, Fernando Goulart y Tulla, Antoni Francesc (2013). «Rota turística da maçã e da sidra no Principado das Astúrias, Espanha». Peniche: VI International Tourism Congress.

Santos, Milton (1996). A natureza do espaço: técnica e tempo, razão e emoção. São Paulo: Hucitec, 2006.

Schneider, Sérgio (2010). «Situando o desenvolvimento rural no Brasil: o contexto e as questóes em debate». Economia Política, 30 (119), 511-531. <http://dx.doi.org/10.1590/S0101-31572010000300009>

SEBRAE (2013). Guia de implementação de indicaçôes geográficas para produtos: orientaçôes para o desenvolvimento de projetos para o reconhecimento de uma indicação geográfica no INPI. Brasília: SEBRAE.

SEBRAE. <http://www.sebrae.com.br> [consulta: 16 de abril de 2014].

SINDIFRANCA. <http://www.sindifranca.org.br> [consulta: 17 de abril de 2014].

Teuber, Ramona (2011). "Consumers' and producers' expectations towards geographical indications: empirical evidence for a german case study». British Food Journal, 113 (7), 900-918. <http://dx.doi.org/10.1108/00070701111148423>

Tortorelli, Cristina Errázuriz (2010). «Indicaciones geográficas y denominaciones de origen». Revista Chilena de Derecho, 37 (2), 207-239.

Valente, Maria Emília Rodrigues; Perez, Ronaldo; Ramos, Afonso Mota y Chaves, José Benício Paes (2012). «Indicação geográfica de alimentos e bebidas no Brasil e na União Europeia». Ciência Rural, 42 (3), 551-558. <http://dx.doi.org/10.1590/S0103-84782012000300027>

Van Ittersum, Koert; Meulenberg, Matthew T. G.; Van Trijp, Hans C. M. y Candel, Math, J. J. M. (2007). "Consumers' appreciation of regional certification labels: a Pan-European study». Journal of Agricultural Economics, 58 (1), 1-23. <http://dx.doi.org/10.1111/j.1477-9552.2007.00080.x>

VARGAS, Ivens Cristian Silva (2008). Indicaçôes geográficas do Brasil: possibilidades para os produtos inseridos na área de proteção ambiental do Ibirapuitã, RS. Tesis de máster no publicada. Santa Maria: Universidade Federal de Santa Maria.

Velloso, Carolina Quiumento (2010). «Elaboração de regulamento de uso, conselho de regulador e definição do controle» En: Cerda, Claire Marie Thuillier (ed.). Curso de propriedade intelectual e inovação no agronegócio: indicação geográfica. Brasília: MAPA.

WIPO. <http://www.wipo.int/geo_indications/en/> [consulta: 7 de agosto de 2013]. 